\title{
A Lifecycle approach to evaluating MyArtSpace
}

\author{
Giasemi Vavoula ${ }^{1}$, Julia Meek ${ }^{2}$, Mike Sharples ${ }^{3}$, Peter Lonsdale ${ }^{2}$, Paul Rudman ${ }^{4}$ \\ ${ }^{1}$ The Open University, ${ }^{2}$ University of Birmingham, ${ }^{3}$ University of Nottingham, ${ }^{4}$ Oxford \\ Brookes University
}

\begin{abstract}
MyArtSpace is a service on mobile phones for enquiry-led museum learning. It enables students to create their own interpretations of museum visits through descriptions of objects, images and sounds. These are automatically transmitted to a personal online gallery that they can use to reflect upon and share their experience. This paper describes the Lifecycle evaluations of MyArtSpace, throughout the design and deployment stages.
\end{abstract}

\section{1: Introduction}

MyArtSpace is a service on mobile phones for enquiry-led museum learning, which also incorporates a web portal for follow up learning after the museum visit. It builds on research by ourselves and others into learning with mobile technologies in museums, in particular to provide links between museum experience and post-visit learning and reflection [1]. The service is currently undergoing trials in three UK museums: the Urbis museum of urban life in Manchester, the D-Day museum in Portsmouth, and the Study Gallery in Poole. Although it can be used for informal learning in museums, the service is particularly suited to school field trips. Before the visit, the teacher will typically set an open-ended question that the students can answer by gathering and selecting evidence from the museum visit. For example, for a visit by Key Stage 3 (US Grade 6-8) students on a history class to the D-Day Museum (a museum to interpret the Allied landings during World War II), the task was to collect evidence to assess whether D-Day was a triumph or a disaster for Britain.

At the museum, students are given Nokia 6680 multimedia mobile phones and each student registers a personal identifier. They can 'collect' an exhibit by typing its two-letter code into the handset, which then displays a multimedia presentation and automatically sends an image and description of the exhibit to their personal collection area in a Web portal (see Figure 1a and $1 \mathrm{~b}$ ). The students are prompted to type in their reasons for collecting, encouraging them to reflect on what they see in the museum in relation to the learning task. After collecting an object, the students are notified of who else has collected it and encouraged to find and talk with them face-to-face. In addition, the students can use the mobile phones to create their own interpretation by taking photos, recording sounds, or writing text comments (see Figure 1c and 1d). This content is also transmitted by the phone to their online collection.

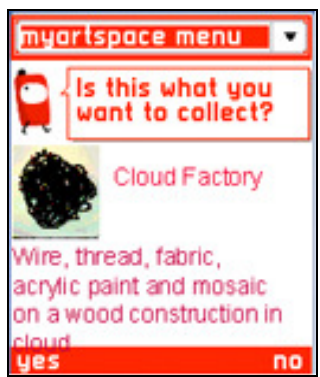

(a)

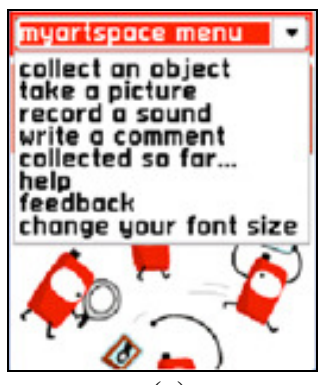

(c)

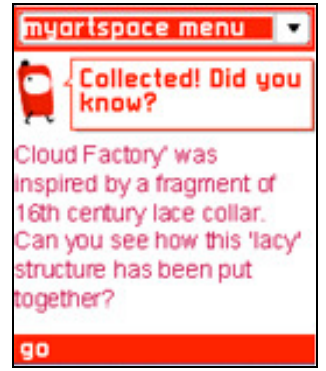

(b)

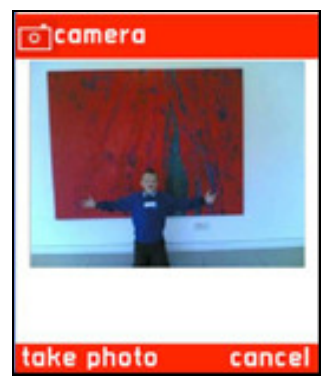

(d)
Figure 1. MyArtSpace mobile phone interface: (a) collecting an object, (b) multimedia presentation about collected object, (c) main menu options, (d) taking photos 
Back at school, the students can view their personal collections, the items collected by the whole class, as well as content made available by the museum. Through a web portal, the students can organize these collections into personal galleries (like simple webbased presentations), to present in the classroom or to share with their family (see Figure 2). Access to the web portal is password protected, and the content published by the students is moderated to ensure privacy protection and appropriate use.

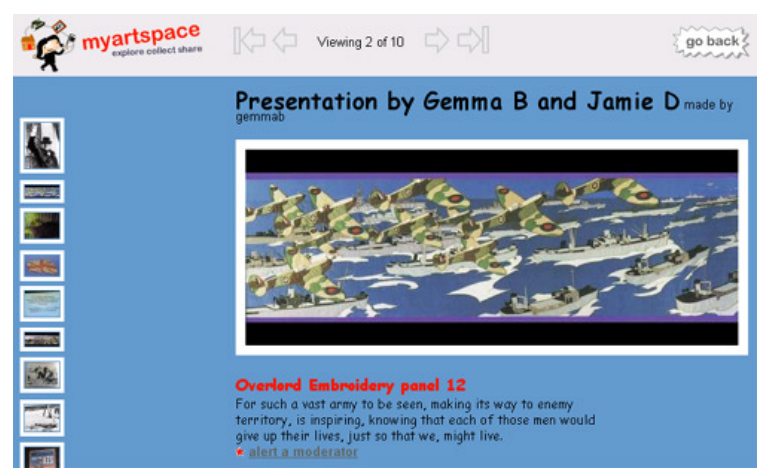

Figure 2. An example student gallery

MyArtSpace was designed and developed by The Sea (www.the-sea.com) and funded by Culture Online, part of the UK Department of Culture, Media and Sports. The user experience and educational evaluation of MyArtSpace was carried out by a team of evaluators at the Centre for Educational Technology and Distance Learning at the University of Birmingham, and was done in parallel with internal testing carried out by the developers. This paper describes the evaluation activities that were undertaken during the development and deployment of MyArtSpace, focusing on the Lifecycle approach to evaluation that was adopted and the user and educational evaluation activities.

\section{2: The Lifecycle Evaluation Approach}

The Lifecycle Evaluation Approach [2][3] places evaluation at the centre of the development process, from the very early stages of design to the final assessment of the technology. The approach draws on evaluation methods and ideas from software engineering, educational evaluation, and models for evaluating learning technology. Evaluation activities are undertaken at key points in the lifecycle of the system design process. The outcomes of each evaluation inform the next stage of the system development or feed into an iteration of an earlier stage. For example, the outcomes of an evaluation during initial user trials inform decisions on how to deploy the system and support the users.

To retain focus in planning and conducting each evaluation, key questions are addressed throughout:

- Goals: Why is the evaluation being undertaken (its purposes and goals)?

- Stakeholders: Who are the stakeholders and the users?

- Timetable: When should the evaluation activities be undertaken?

- Deliverables: How will the results be communicated?

- Methods: What evaluation methods and resources will be used?

These questions enable the evaluation team to retain focus on the task. For MyArtSpace, the stakeholders included the funding body, the development team, teachers, LEA (local education authority) representatives, and museum curators and educational experts. The overall goal of the evaluation was agreed by the key stakeholders to be the assessment of the service's usability, accessibility, effectiveness, and capability to engage the user. This broad goal was expanded into a set of requirements following a user consultation workshop that brought together representatives from the key stakeholder groups. The timetable, deliverables and evaluation methods were subsequently designed and agreed with respect to the identified requirements.

\section{3: Compiling and validating requirements}

The requirements (112 in total) that emerged from the user consultation workshop were divided into six main categories:

1. Teacher requirements: requirements for designing the teacher experience

2. Student requirements: requirements for designing the student experience

3. Museum requirements: requirements for designing the museum staff experience and also institutional requirements

4. Culture Online requirements: requirements relevant to the funding body, pertaining to overall project objectives, qualitative and quantitative measurement criteria, etc.

5. General system requirements: all other requirements that did not directly concern a specific group

6. Usability requirements: requirements for designing an easy to use, easy to learn and enjoyable service/system for all user groups

Requirements identified in the above categories were further characterised as educational (E) or user 
(U). Educational requirements related to the educational experience whereas user requirements concerned functionality and performance.

One of the key features of the Lifecycle Evaluation Approach is the early and continual involvement of stakeholders in the evaluation process in order to agree priorities and agendas. The initial set of requirements was validated and prioritised through a further Stakeholder Workshop, which brought together 16 representatives from the main stakeholder groups. . The stakeholders who participated in the consultation workshop followed through the evaluation process and were involved in the pilot studies.

Due to the number of requirements that had been generated, the representatives were divided into groups and each group was given a subset of requirements to review and was asked to verify that each requirement was unambiguous and testable. Subsequently, the stakeholders were asked to use the MoSCoW [4] technique from Dynamic Systems Development Method to indicate that a requirement is a:

- MUST: must have this

- SHOULD: should have this if at all possible

- COULD: could have this if it does not affect anything else

- WOULD: won't have this time but would like to have in the future

The outcomes of the discussion in the subgroups were then presented to the larger group, who finalised the categorisation and prioritisation.

The workshop was successful in enabling the requirements to be discussed, clarified and prioritised. The outcome was an agreed set of user requirements and benchmarks for both the Web portal of MyArtSpace and the Mobile phone service.

An additional benefit of the workshop was the stakeholders' increased involvement, enthusiasm and ownership of MyArtSpace through their participation in the design of the evaluation process.

Evaluation activities were subsequently planned to test the identified user and educational requirements. The user requirements were tested in two stages: through heuristic evaluations that were dispersed throughout the system design phase, and through onsite user observations that took place during pilot deployment. The educational requirements were tested during pilot deployment.

\section{4: Evaluation of of educational and user requirements during the design phase}

A series of evaluations focusing on usability were carried out during the system design phase. Formative evaluations were carried out on paper based prototypes of both components of the system (mobile phone service interface and web portal).

The main method utilized was Heuristic Evaluation, a 'discount usability' method developed by Nielsen [5] and widely used in usability evaluations of websites and interactive software. The method is particularly effective in finding and prioritising specific usability problems [6]. It employs a group of evaluators (three to five is recommended) with expertise in humancomputer interaction. Each evaluator separately follows a scenario to interact with the system while referring to a set of specified usability principles (the 'heuristics'). Subsequently, with the assistance of a recorder, the evaluator produces a written $\log$ of incidents where the system violates the heuristics. Together with the recorder, the evaluator allocates a severity rating to each of these usability problems. The outcome is a report listing the main usability problems indicating, for each, its context within a scenario of use and its severity.

The heuristic evaluations of the paper based prototypes highlighted important usability issues on both the mobile phone service interface and the web portal for teachers, students and museum staff. For example, problems with the main menu of the mobile phone interface were identified (the names of the menu items are unclear). These usability issues, especially the ones with high severity ratings, were rectified in subsequent versions of the system components, ensuring that they do not propagate into the fully developed MyArtSpace service. This is one of the benefits of adopting a Lifecycle approach to evaluation; problems are identified at an early stage when they can be easily corrected, whereas changing a product after deployment can be costly and not always possible.

\section{5: Evaluation of educational and user requirements during pilot deployment}

Two detailed pilot studies were conducted, one at the Urbis museum in Manchester, UK, in November 2005 with Key Stage 2 students; and one at the D-Day museum in Portsmouth, UK, in January 2006 with Key Stage 3 students. The studies were designed in two phases to enable further refinement of the evaluation methods and instruments.

As described in the introduction, the learning experience with MyArtSpace includes three stages: the first stage (preparation) takes place in the classroom, where with the optional aid of the web portal the teacher prepares the class for the museum visit; the 
second stage (museum visit) takes place at the museum, where the students explore the exhibits and use the mobile phones to collect museum items and to create their own content (photos, audio notes, text notes); the third stage (post museum visit) takes place back in the classroom, where the students use the web portal to edit, present and share their personal galleries. Evaluation activities were designed for each stage and included:

\section{1:Preparation}

Pre $1^{\text {st }}$ School Lesson: a telephone interview with the teacher before the preparation lesson focused on the learning outcomes against which the teacher would judge the success of the experience. The aim of the interview was also to find out how the teacher planned to run the preparation, museum visit, and post-visit activities, as well as to make all admin arrangements (including permissions to carry out evaluation activities).

$1^{\text {st }}$ School Lesson: observations of the teacher and the students during the first school lesson focused on finding out how both teacher and students work around the MyArtSpace portal, whether they have any difficulties in understanding and using it, how the whole service is introduced by the teacher and how the learning activities to follow are introduced.

Post $1^{\text {st }}$ School Lesson: an interview with the teacher and two focus groups with students focused on their impressions from the preparation lesson: did it differ to other museum visit preparations and how? How did the preparation lesson shape their expectations for the museum visit? Did they have any particular problems in using the web portal for introduction purposes?

\section{2: Museum visit}

Pre museum visit: a structured interview with museum staff focused on their expectations from the introduction of MyArtSpace, the identification of operating costs generated by it, and also the identification of added value for the visitor experience.

Museum visit: observations of teachers, students and participating museum staff focused on how the service is used in practice and how the teachers and museum staff assist, direct and facilitate the student experience in relation to the use of the mobile phone service. Also usability problems were identified and noted.

Post museum visit: structured interviews with museum staff focused on their perceptions and opinions about the museum adopting MyArtSpace. Interviews with teachers focused on their impressions about
MyArtSpace-enabled school visits to museums and how it enhances/hinders the learning process, and also to highlight problems experienced during the visit. Finally, focus groups with students sought to find out their impressions from using the MyArtSpace mobile phone service, whether and how it enhanced their visit, and what problems they had in using it.

\section{3: Post Museum Visit}

$2^{\text {nd }}$ School Lesson: observations of the teacher and students during the second school lesson that followed the museum visit, focused on how they work with the MyArtSpace portal.

Post $2^{\text {nd }}$ School Lesson: a structured interview with the teachers and focus groups with the students focused on how the lesson differed to other post museum visit lessons, whether they had any problems is using the MyArtSpace portal, what advantages/disadvantages they perceived in using it, and whether their expectations for the MyArtSpace experience were fulfilled, what were their general impressions, and whether they would use it again. The students in particular were asked whether they thought the service was fun to use, whether they thought they learned more than during traditional museum visits, and whether they planned to share their personal galleries with friends and family.

\section{6: Discussion}

The first pilot study at the Urbis museum highlighted several important issues, both in terms of system functionality and performance and in terms of the way the system is integrated in the classroom. The second pilot study took place two months later, allowing time for the evaluation results to be communicated to the development team who in turn revised the system accordingly. This trial verified that the majority of the performance problems that occurred in the first trial were resolved, allowing the evaluation to identify more detailed and specific usability problems and to further clarify how best to integrate the service with school practices.

The severe system performance problems identified during the first full-scale trial could not have been easily identified with small numbers of users, e.g. during internal testing. Challenging as it may be to bring a design to a working level enough to support realistic trials while remaining flexible enough to be able to accommodate changes, at the same time it is necessary if performance is to be tested alongside functionality.

The first pilot study highlighted that the students were very keen on using the phones to take 
photographs, without necessarily entering a description of the object being photographed and explaining their reason for collecting. They did not appreciate that the notes made during the collection phase in the museum would later assist them in creating their MyArtSpace web gallery from their collected items. This problem could be resolved with additional training, to make students aware of the importance of carefully selecting the objects to collect and of recording their responses to them; it also highlights the need for students to realise not only what the technology can do, but also to appreciate how this can be educationally useful and relevant to them.

Extended use of the photo function was also made by students in the second study. The learning task in the second study asked the students to explore the museum and collect objects while trying to form a view based on the contents of the museum (whether D-Day was a triumph or a disaster, as described in the introduction). Since the students had not decided on a viewpoint in advance, they tended to collect objects irrespectively of which view they supported in the end. This also contributed towards a vast collection of objects, which was later hard to manage, manipulate and deconstruct. This observation draws attention to the fact that care is needed not only in designing the technology, but also in designing the learning task that makes use of the technology. Our recommendation was that teachers should be allowed to customise MyArtSpace by adding content specific to their lesson plans.

Our evaluations of the service have collected positive feedback from students, teachers, Local Education Authority representatives and museum educators.

"The day was of tremendous benefit to the pupils and their history studies. The mobile phones were easy to use and the children were quickly off exploring the museum and making their own collections. I have not seen pupils so engaged or enthusiastic on a museum visit before." (Teacher, D-Day museum)

The way the 'collection' of museum items takes place encourages students to stop and think about each exhibit: what is the exhibit about, and how does it relate to their learning task?

"Made me look at artwork more ... Most people think going to galleries is boring, but when you put ideas on a web site and use the phones it's much more fun." (Student, The Study Gallery)

\section{7: Conclusions}

The Lifecycle Evaluation Approach placed the focus on evaluation from the very start of the project. Stakeholders were involved throughout, making the evaluation 'everyone's business' and ensuring that findings feed into the design and development process. The early identification of problems and issues during carefully spaced evaluation activities enabled them to be resolved prior to further trials and prior to the system being widely deployed.

MyArtSpace is a service that makes use of different technologies (mobile phones and the Web) to deliver a learning experience that traverses the museum and the classroom. It was therefore essential that the evaluation of the service did not stop at the evaluations of its individual components; rather, it had to go beyond the technology to look at the learning experience as a whole. The Lifecycle Evaluation Approach has made this possible through its focus on both the usability and the pedagogy of MyArtSpace, which enabled a full picture of the use of a new educational technology to be understood, assessed and improved.

\section{8: Acknowledgements}

We would like to thank all the students and teachers who enthusiastically took part in the evaluations and showed us the potential of MyArtSpace. We would also like to thank all those who participated in the user consultation workshops, giving up their time to inform the evaluations of the service.

\section{9: References}

[1] Mulholland, P., Collins, T. \& Zdrahal, Z. (2005) Bletchley Park Text: Using mobile and semantic web technologies to support the post-visit use of online museum resources. Journal of Interactive Media in Education [jime.open.ac.uk/2005/24<http://jime.open.ac.uk/2005/24/>].

[2] Meek, J. (2006) Adopting a Lifecycle Approach to the Evaluation of Computers and Information Technology. Unpublished PhD Thesis, The University of Birmingham, UK.

[3] Meek, J. and Sharples, M. (2001) A lifecycle approach to the evaluation of learning technology. Proceedings of CAL2001 Conference, Warwick, 2-1 April, pp. 195-196.

[4] http://www.dsdm.org/

[5] Nielsen, J. (1993). Usability Engineering, Academic Press, Inc.

[6] Nielsen J (1994). How to Conduct a Heuristic Evaluation, [online], http://useit.com/papers/heuristic /heuristic_evaluation.html 